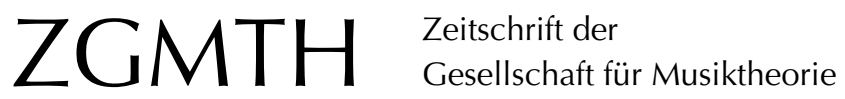

Jans, Markus (2006): Sieben (nicht ganz wahllose) Griffe in die Bücherkiste. ZGMTH 3/1, 145-148. https://doi.org/10.31751/213

(C) 2006 Markus Jans

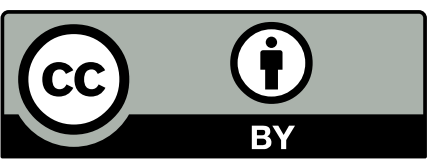

Dieser Text erscheint im Open Access und ist lizenziert unter einer Creative Commons Namensnennung 4.0 International Lizenz.

This is an open access article licensed under a

Creative Commons Attribution 4.0 International License.

veröffentlicht / first published: 01/01/2006

zuletzt geändert / last updated: 01/12/2008 


\section{Sieben (nicht ganz wahllose) Griffe in die Bücherkiste}

Markus Jans

1. Humberto R. Maturana / Francisco J. Varela, Der Baum der Erkenntnis, Wie wir die Welt durch unsere Wahrnehmung erschaffen - die biologischen Wurzeln der menschlichen Erkenntnis, aus dem Spanischen übersetzt von Kurt Ludewig, Bern/München/Wien: Scherz 1987.

Zwei führende Neurobiologen stellen in einem Lehrbuch ihre Forschungsergebnisse über elementare Lebensprozesse vor. Ihr Hauptinteresse gilt den Wahrnehmungsvorgängen, über die wir zu einer (wie auch immer gearteten) Vorstellung von Wirklichkeit kommen. Mit den Untersuchungsmethoden der Naturwissenschaft gelangen sie zu Schlüssen, die vergleichbar sind mit Ideen, die in der Geschichte der philosophischen wie auch der esoterischen Epistemologie immer wieder eine Rolle gespielt haben: Wahrnehmen ist Wirklichkeit erschaffen. Subjekt und Objekt sind Aspekte dieser Wirklichkeit und eigentlich nicht voneinander zu trennen. Das verwendete wissenschaftliche Vorgehen zur Wahrheitsfindung lässt sich selbstverständlich problematisieren und wird gerade durch die Ergebnisse dieser Studie ganz schön relativiert. Die Autoren wissen daraus brisantes Kapital zu schlagen.

2. Luc Ciompi, Die emotionalen Grundlagen des Denkens. Entwurf einer fraktalen Affektlogik, Göttingen: Vandenhoeck 1997.

Der Schweizer Psychiater und Kognitionsforscher bricht mit der Vorstellung einer kausalen Logik für Denken und Handeln. Letztere unterstellt, dass sowohl Denken als auch Handeln einer kausalen Folgerichtigkeit unterstehen, desgleichen die Interaktion zwischen Denken und Handeln. Das untrennbare Ineinander von Kognition und Emotion hebelt nach Ansicht Ciompis die obgenannte Kausalverkettung aus. Sowohl Denken als auch Handeln sind weder stringent rückführbar auf Ursächlichkeiten, noch sind sie sinnvoll als Verkettung von solchen darzustellen. Mit der sSelbstähnlichkeits, einem Begriff, der in der Chaostheorie eine bedeutsame Rolle spielt, wird ein anderer möglicher ১Ordnungsfaktor`vorgeschlagen und eingeführt. Denken und Handeln sind eingebunden in einen »unablässigen Prozess der Selbstschöpfung [...], die sich mit ihr eigener Kraft wieder und wieder selbstähnlich hervorzubringen vermag«. Per saldo: Kein Gedanke ohne Gefühl in seinem `Vorraum», das aber heißt: „Addio reine ratio!« 
3. Heinz von Foerster, KybernEthik, Berlin: Merve 1993.

Der Autor ist einer der Begründer und wichtigsten Vertreter der in den 40er Jahren des 20. Jahrhunderts entstandenen Wissenschaft von der Steuerung von Ereignissen und Abläufen. Er erzählt in diesem Buch über sein Leben, über prägende Begegnungen, über seine Wissenschaft und ihren Beitrag zur Philosophie, insbesondere zu Erkenntnistheorie und Ethik. Abgedruckt sind mehrere Reden und Aufsätze zu Haupt- und Nebenaspekten des Faches, die Heinz von Foerster vor ganz unterschiedlichen Gremien in den USA und in Europa gehalten und später publiziert hat. Neben einer Einführung in die natürliche Magie findet sich da sogar eine Einführung in die 12-Ton Musik. Foerster war bekannt mit Josef Maria Hauer. Er berichtet zunächst von einer Begegnung mit dem Komponisten, die er als Adoleszent erlebt hat, und fügt dieser Geschichte einen Aufsatz an, den er für die erste Nummer der Zeitschrift Jedermann 1947 zum Thema geschrieben hatte. Die Hauptstücke des Buches betreffen allerdings weniger die technischen als vielmehr die philosophischen und sozialen Implikationen, genauer: den Einfluss, den die relativ junge Wissenschaft mit ihrer durch Rückkoppelung und Vernetzung geprägten Sicht auf die Erkenntnistheorie und auf die Ethik haben kann. Zur Debatte steht ein Paradigmenwechsel, darin die Abkehr von der Idee (und Denkpraxis) der Objektivität die Hauptrolle spielt. Zur Diskussion steht der Übergang vom linear gerichteten (am ehesten durch das Symbol des Pfeils repräsentierten) zu einem gleichzeitig mehrfach orientierten (vielleicht durch ein kugelförmiges Netz am besten symbolisierten) Selbst- und Weltbewusstsein, oder, um es mit Jean Gebser (Ursprung und Gegenwart, 1949) zu sagen: der Übergang vom perspektivischen zum aperspektivischen Bewusstsein. Angesichts der wachsenden individuellen Freiheit steigt die Bedeutung der Wahl und damit das Gewicht der persönlichen (und kollektiven) Verantwortung. Hier ergibt sich die Verbindung zur Ethik, die im Buchtitel mit der Kybernetik kurzgeschlossen wird.

4. Guy André Mayor, Anonymität und Oeffentlichkeit. Eine textlinguistische Untersuchung und Beschreibung der Rubrik "Schatzchäschtli« des Zürcher TAGES-ANZEIGERs, ihrer Texte und Textsorten von 1978 bis 1998, Luzern: ars pro toto 2002.

Mayor interessiert sich in diesem Buch für das sprachliche Handeln in Kleinannoncen, für die möglichen Vorder- und Hintergründe von öffentlicher Intimität und gleichzeitig anonymer Öffentlichkeit. Die Auslegeordnung verschiedener Textsorten und besonders die damit verbundene Verfolgung einzelner `Geschichten über beträchtliche Zeiträume lesen sich bisweilen wie Krimis. Über das Einordnen und Situieren der Texte geraten mehr und mehr auch die dazu unabdingbaren Deutungen ins Blickfeld: Was ist gesagt, was ist gemeint? Wie nehmen wir Text und Subtext wahr? Und hier, im Bereich der Rezeption und Interpretation von Texten, bietet Mayor (auch für Musiktheoretiker) neue und sehr hilfreiche Denkansätze: Er stellt, erstmals in der Linguistik und Literaturwissenschaft, ein senergetisches« Text- und Textverstehensmodell zur Diskussion, das sich sehr gut auch auf musikalische Texte/Werke übertragen lässt. Ich habe dieses Modell kürzlich in einem Aufsatz über Interpretation (Musiktheorie 4, 2005) vorgestellt. Ich denke, dass eine (dort ebenfalls zitierte) Textpassage genügt, um zu erklären, wie dieses senergetisch 
zu verstehen ist: „Darüber hinaus enthalten Texte - neben linguistisch kategorisierbaren Elementen und physikalisch messbaren Schwingungen (etwa Schallwellen) - Schwingungsmuster, die mit letzteren auf eine noch nicht durchschaubare Weise verbunden sind. Texte sind also, meiner These entsprechend, immer auch ıSchwingungsfelder bzw. deren (oben genannte) diskriminierbare Teile vielleicht `Träger einer feinstofflichen, apparativ (noch) nicht messbaren Energie, die ich, in Anlehnung an Sheldrake, morphogenetische nenne.« Die Modellvorstellung von morphischen bzw. morphogenetischen Energiefeldern wurde von Rupert Sheldrake, einem Biologen, eingeführt (Das schöpferische Universum, 1983), um gewisse zwar nachweisbare, aber in ihrer Funktionsweise unerklärliche Informationsübertragungen darstellen zu können. Das Feldmodell scheint besonders treffend, denn auch bei den aus der Physik bekannten Feldern ist deren Vorhandensein bloß an ihrer Wirkung erfahr- und messbar.

5. Max Haas, Musikalisches Denken im Mittelalter. Eine Einführung, Bern u.a.: Peter Lang 2005.

Der Basler Musikologe legt ein Fazit seiner lebenslangen Mittelalterforschung vor. Seine Darstellung erfasst und deutet nicht nur alle verfügbaren Quellen (und deren Quellen und deren historische Kommentare), sondern verarbeitet auch eine atemberaubende Zahl von themenverwandter und sachrelevanter Sekundärliteratur. Die große Menge an Information verlangt nach einer bekömmlichen Form der Darreichung. Sie wurde gefunden in der Fokussierung auf einige Kernthemata, auf die bezogen der Text sich ausbreitet (und Seitenthemen entwickelt), um die herum er mäandriert, und auf die er immer wieder von anderen Betrachtungsstandpunkten aus zugeht und zurückkommt. Sehr viele unserer mehr oder minder zementierten Ansichten zum Mittelalter und zum mittelalterlichen Musikdenken werden dabei in ihren Grundfesten erschüttert. Haas macht sich und dem Leser die Sache nicht einfach. Jeder einzelne Begriff, jede einzelne Vorstellung wird mit rigoroser Gründlichkeit hinterfragt, kein Stein bleibt auf dem anderen. Ob es um die Voraussetzungen des mittelalterlichen Denkens überhaupt und um den mächtigen Einfluss von Aristoteles und dessen Rezeption, ob es um das suniversitäre Curriculum` (auf Papier und in der Praxis), ob es um das musikalische Handeln (operari) und die damit verbundene Vorstellung von Werk (opus), ob es um die Sprache von Musiktraktaten und um die Hintergründe ihrer Verfasstheit, oder ob es (in diesem Zusammenhang) um deren Adressaten (Kinder und Adoleszenten) geht: Nichts bleibt ganz so und nichts bleibt so ganz, wie wir es bisher geglaubt haben. Die Wellen der Erschütterung machen zudem nicht etwa halt an den Grenzen (wo wären diese zu ziehen?) des Mittelalters, sondern breiten sich mit voller Stärke aus bis ins 17. Jahrhundert.

6. Christopher Schmidt, Harmonia Modorum. Eine Gregorianische Melodielehre, Winterthur: Amadeus 2004.

Schmidt führt in die Tiefe von Modus und Modalität und zeigt an seinen (notwendigerweise) sehr vielen Beispielen das Ineinander aller Modi so überzeugend auf, dass sämtliche alten und neuen Bestimmungskriterien und Erfassungssysteme sich daneben ärmlich ausnehmen. In meiner Buchbesprechung in dieser Ausgabe ist dazu mehr zu erfahren. 
7. A. \& N. O’Nymi: Die romantische Harmonielehre und ihre Krise (nicht nur) in Wagners Tristan (... sondern auch bei Mozart, Bach, Mahler und vielen anderen, ja eigentlich bei allen). Systematische Theorie und ihre hausgemachten Probleme, Verlag »Herr, es ist Zeit», erschienen im Jahre Zweitausendundhoffentlichbald.

Ich weiß: Ludwig Holtmeier hat die Büchse der Pandora bereits ganz nett geöffnet (Dresden 2001). Die (nicht zuletzt) dadurch um sich greifende Epidemie bedarf m. E. eines finalen Schubs, der sie zur Pandemie werden lässt. Ein Buch, das ich gerne lesen würde. 